\title{
Discrete 2D Analytic Image Decomposition
}

\author{
Xu Guanlei, a , Wang Xiaotong ${ }^{2, b}$ and Xu Xiaogang ${ }^{3, c}$ \\ ${ }^{1}$ Ocean department of Dalian Naval Academy, Dalian, China, 116018 \\ ${ }^{2,3}$ Automation and navigation department of Dalian Naval Academy, Dalian, China, 116018 \\ axgl_86@163.com, bhjdljtxy@163.com, ${ }^{c}$ xxgang@cad.zju.edu.cn
}

Keywords: Discrete Hilbert transform (DHT); Bedrosian's theorem.

\begin{abstract}
In this paper, we first investigate the discrete 2D analytic signals with some properties such as Discrete Bedrosian's theorems. Second, based on the derived discrete properties of discrete 2D analytic signals, we provide an image decomposition method via the above principles to decompose image. Experiments on texture analysis show the efficiency of the proposed methods.
\end{abstract}

\section{Introduction}

Hilbert transform plays an important role in signal processing, mathematics and et al.[1-5]. Hilbert transform and the associated concept of analytic signals, introduced by Gabor [1], have been widely adopted for time-frequency analysis in diverse applications of signal processing. In this paper, we will discuss the 2D discrete-spatial signals. Discrete-spatial signals (images) are represented mathematically as sequences of numbers. In continuous space, for $2 \mathrm{D}$ signal processing, the classical HT can mainly be divided into the follows: total HT(THT), partial HT(PHT), single orthant HT(SOHT) and so on[3-5]. In this paper we will mainly discuss the three types of HT: PHT, THT and SOHT with their properties.

\section{Discrete 2D Hilbert Transform and Analytic Signals}

We consider the sequence for which the Fourier transforms are zero on $-\pi \leq \omega_{x}<0$. Then, with $z(m, n)$ denoting the sequence and $Z\left(e^{i \omega_{x}}, e^{i \omega_{y}}\right)$ its Fourier transform, we require that

$$
Z\left(e^{i \omega_{x}}, e^{i \omega_{y}}\right)=0,-\pi \leq \omega_{x}<0 .
$$

The sequence $z(m, n)$ corresponding to $Z\left(e^{i \omega_{x}}, e^{i \omega_{y}}\right)$ must be complex. Therefore, we can express $z(m, n)$ as follows: $z(m, n)=z_{R}(m, n)+i z_{I}(m, n)$,

where $z_{R}(m, n)$ and $z_{I}(m, n)$ are real sequences (real part and imaginary part, respectively). If $Z_{R}\left(e^{i \omega_{x}}, e^{i \omega_{y}}\right)$ and $Z_{I}\left(e^{i \omega_{x}}, e^{i \omega_{y}}\right)$ denote the Fourier transform of the real sequence $z_{R}(m, n)$ and $z_{I}(m, n)$, respectively, then $Z\left(e^{i \omega_{x}}, e^{i \omega_{y}}\right)=Z_{R}\left(e^{i \omega_{x}}, e^{i \omega_{y}}\right)+i Z_{I}\left(e^{i \omega_{x}}, e^{i \omega_{y}}\right)$.

In addition, we also have $Z_{I}\left(e^{i \omega_{x}}, e^{i \omega_{y}}\right)=-i \operatorname{sgn}\left(\omega_{x}\right) Z_{R}\left(e^{i \omega_{x}}, e^{i \omega_{y}}\right)=H_{x}\left(e^{i \omega_{x}}, e^{i \omega_{y}}\right) Z_{R}\left(e^{i \omega_{x}}, e^{i \omega_{y}}\right)$,

where $H_{x}\left(e^{i \omega_{x}}, e^{i \omega_{y}}\right)=-i \operatorname{sgn}\left(\omega_{x}\right)$ and $\operatorname{sgn}(\omega)=\left\{\begin{array}{ll}+1, & \text { for } 0<\omega<\pi \\ 0, & \text { for } \omega=0 \\ -1, & \text { for }-\pi<\omega<0\end{array}\right.$ is the signum function.

Also, we have $h_{x}(m, n)=\frac{1}{2 \pi} \int_{-\pi}^{\pi} \int_{-\pi}^{\pi} H_{x}\left(e^{i \omega_{x}}, e^{i \omega_{y}}\right) e^{i\left(\omega_{x} m+\omega_{y} n\right)} d \omega_{x} d \omega_{y}$

$$
=\left\{\begin{array}{ll}
\frac{2}{\pi} \frac{\sin ^{2}(\pi m / 2)}{m} \delta(n), & m \neq 0 \\
0, & m=0
\end{array} .\right.
$$

In spatial domain, we let $H_{x}^{d}$ denote the discrete partial Hilbert transform operator along $\mathrm{X}$ axis, and take into account the relation of triangle, we can obtain 
$H_{x}^{d}\{z(m, n)\}=\sum_{l \in \mathbb{Z}} h_{x}(m-l, n) z(l, n)=\frac{1}{\pi} \sum_{l \in \mathbb{Z}}\left\{\frac{1-\cos \pi(m-l)}{m-l}\right\} z(l, n)= \begin{cases}\frac{2}{\pi} \sum_{l \text { odd }} \frac{z(l, n)}{m-l}, & \text { for even } m \\ \frac{2}{\pi} \sum_{l \text { even }} \frac{z(l, n)}{m-l}, & \text { for odd } m \\ 0, & \text { others }\end{cases}$

The inverse relationship is given by $z(m, n)= \begin{cases}-\frac{2}{\pi} \sum_{m \text { odd }} \frac{H_{x}^{d}\{z(m, n)\}}{l-m}, & \text { for even } l \text {, } \\ -\frac{2}{\pi} \sum_{m \text { even }} \frac{H_{x}^{d}\{z(m, n)\}}{l-m}, & \text { for old } l .\end{cases}$

At the same time, we can also deduce that $\left(H_{x}^{d}\right)^{-1}=-H_{x}^{d}$ and $\left(H_{x}^{d}\right)^{2}=-I$, where $I$ is the identity operator. In the same manner, we can obtain the similar forms for the partial Hilbert transform along $\mathrm{Y}$ axis that are trivial and will not be shown here. Similarly, for the total Hilbert transform, we consider the sequence for which the Fourier transforms are non-zero on $0<\omega_{x} \leq \pi$ and $0<\omega_{y} \leq \pi$.

Then, with $z(m, n)$ denoting the sequence and $Z\left(e^{i \omega_{x}}, e^{i \omega_{y}}\right)$ its Fourier transform, we require that

$$
\left\{\begin{array}{ll}
Z\left(e^{i \omega_{x}}, e^{i \omega_{y}}\right) \neq 0, & \text { for }\left(0<\omega_{x} \leq \pi\right) \cap\left(0<\omega_{y} \leq \pi\right) \\
Z\left(e^{i \omega_{x}}, e^{i \omega_{y}}\right)=0, & \text { for others }
\end{array} .\right.
$$

The sequence $z(m, n)$ corresponding to $Z\left(e^{i \omega_{x}}, e^{i \omega_{y}}\right)$ must be complex. Therefore, we can express $z(m, n)$ as follows: $z(m, n)=z_{R}(m, n)+i z_{I}(m, n)$, where $z_{R}(m, n)$ and $z_{I}(m, n)$ are real sequences (real part and imaginary part, respectively). If $Z_{R}\left(e^{i \omega_{x}}, e^{i \omega_{y}}\right)$ and $Z_{I}\left(e^{i \omega_{x}}, e^{i \omega_{y}}\right)$ denote the Fourier transform of the real sequence $z_{R}(m, n)$ and $z_{I}(m, n)$, respectively, then $Z\left(e^{i \omega_{x}}, e^{i \omega_{y}}\right)=Z_{R}\left(e^{i \omega_{x}}, e^{i \omega_{y}}\right)+i Z_{I}\left(e^{i \omega_{x}}, e^{i \omega_{y}}\right)$.

In addition, we also have $Z_{I}\left(e^{i \omega_{x}}, e^{i \omega_{y}}\right)=H_{T}\left(e^{i \omega_{x}}, e^{i \omega_{y}}\right) Z_{R}\left(e^{i \omega_{x}}, e^{i \omega_{y}}\right)$,

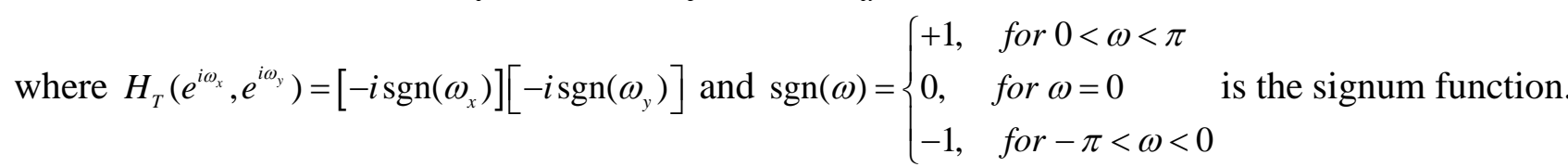

Also, we have

$$
h_{T}(m, n)=\frac{1}{2 \pi} \int_{-\pi}^{\pi} \int_{-\pi}^{\pi} H_{T}\left(e^{i \omega_{x}}, e^{i \omega_{y}}\right) e^{i\left(\omega_{x} m+\omega_{y} n\right)} d \omega_{x} d \omega_{y}=\left\{\begin{array}{ll}
\frac{2}{\pi} \frac{\sin ^{2}(\pi m / 2)}{m} \frac{\sin ^{2}(\pi n / 2)}{n}, & m \neq 0 \bigcap n \neq 0 . \\
0, & \text { others }
\end{array} .\right.
$$

In spatial domain, we let $H_{T}^{d}$ denote the discrete total Hilbert transform operator, and take into account the relation of triangle, we can obtain

$$
H_{T}^{d}\{z(m, n)\}=\sum_{l \in \mathbb{Z}} \sum_{p \in \mathbb{Z}} h_{T}(m-l, n-p) z(l, p)=\left\{\begin{array}{ll}
\frac{2}{\pi} \sum_{l \text { odd }} \sum_{p \text { odd }} \frac{z(l, p)}{(m-l)(n-p)}, & \text { for even } m \text { and even } n \\
\frac{2}{\pi} \sum_{l \text { even }} \sum_{p \text { even }} \frac{z(l, p)}{(m-l)(n-p)}, & \text { for odd } m \text { and odd } n \\
\frac{2}{\pi} \sum_{l \text { odd }} \sum_{p \text { even }} \frac{z(l, p)}{(m-l)(n-p)}, & \text { for even } m \text { and odd } n \\
\frac{2}{\pi} \sum_{l \text { even }} \sum_{p \text { odd }} \frac{z(l, p)}{(m-l)(n-p)}, & \text { for odd } m \text { and even } n \\
0, & \text { others }
\end{array} .\right.
$$

The inverse relationship is given by 


$$
z(m, n)= \begin{cases}-\frac{2}{\pi} \sum_{m \text { odd }} \sum_{n \text { odd }} \frac{H_{T}^{d}\{z(m, n)\}}{(l-m)(p-n)}, & \text { for even l and even } p, \\ -\frac{2}{\pi} \sum_{m \text { even }} \sum_{n \text { even }} \frac{H_{T}^{d}\{z(m, n)\}}{(l-m)(p-n)}, & \text { for odd l and odd } p, \\ -\frac{2}{\pi} \sum_{m \text { even }} \sum_{n \text { odd }} \frac{H_{T}^{d}\{z(m, n)\}}{(l-m)(p-n)}, & \text { for odd l and even } p, \\ -\frac{2}{\pi} \sum_{m \text { odd }} \sum_{n \text { even }} \frac{H_{T}^{d}\{z(m, n)\}}{(l-m)(p-n)}, & \text { for even l and odd } p .\end{cases}
$$

\section{Properties of Discrete 2D Hilbert Transform}

Lemma 1: Let $Z\left(e^{i \omega_{x}}, e^{i \omega_{y}}\right)$ be the discrete-spatial Fourier transform of $z(m, n)$. Then $z(m, n)$ is an analytic sequence iff $Z\left(e^{i \omega_{x}}, e^{i \omega_{y}}\right)=0$ for $-\pi<\omega_{x}<0\left(-\pi<\omega_{y}<0\right)$ in viewpoint of partial Hilbert transform along $\mathrm{X}(\mathrm{Y})$ axis.

Lemma 2: Let $Z\left(e^{i \omega_{x}}, e^{i \omega_{y}}\right)$ be the discrete-spatial Fourier transform of $z(m, n)$. Then $z(m, n)$ is an analytic sequence iff $Z\left(e^{i \omega_{x}}, e^{i \omega_{y}}\right)=0$ for $-\pi<\omega_{x}<0 U-\pi<\omega_{y}<0$ in viewpoint of total Hilbert transform.

Theorem 1: Suppose that $z_{1}(m, n)$ and $z_{2}(m, n)$ are complex sequences with discrete-spatial Fourier transforms $Z_{1}\left(e^{i \omega_{x}}, e^{i \omega_{y}}\right)$ and $Z_{2}\left(e^{i \omega_{x}}, e^{i \omega_{y}}\right)$. Then $H_{x}^{d}\left\{z_{1} z_{2}\right\}(m, n)=z_{1}(m, n) H_{x}^{d}\left\{z_{2}(m, n)\right\}$

if there exists a nonnegative number $\sigma<\pi$ such that

$$
\begin{aligned}
& Z_{1}\left(e^{i \omega_{x}}, e^{i \omega_{y}}\right)=0 \text { for } 0<\sigma<\left|\omega_{x}\right|<\pi \text { and } Z_{2}\left(e^{i \omega_{x}}, e^{i \omega_{y}}\right)=0 \text { for } 0<\left|\omega_{x}\right|<\sigma<\pi \text {, or } \\
& H_{y}^{d}\left\{z_{1} z_{2}\right\}(m, n)=z_{1}(m, n) H_{y}^{d}\left\{z_{2}(m, n)\right\}
\end{aligned}
$$

if there exists a nonnegative number $\sigma<\pi$ such that

$$
Z_{1}\left(e^{i \omega_{x}}, e^{i \omega_{y}}\right)=0 \text { for } 0<\sigma<\left|\omega_{y}\right|<\pi \text { and } Z_{2}\left(e^{i \omega_{x}}, e^{i \omega_{y}}\right)=0 \text { for } 0<\left|\omega_{y}\right|<\sigma<\pi \text {. }
$$

The proof is trivial.

Theorem 2: Suppose that $z_{1}(m, n)$ and $z_{2}(m, n)$ are complex sequences with discrete-spatial Fourier transforms $Z_{1}\left(e^{i \omega_{x}}, e^{i \omega_{y}}\right)$ and $Z_{2}\left(e^{i \omega_{x}}, e^{i \omega_{y}}\right)$. Then $H_{x}^{d}\left\{z_{1} z_{2}\right\}(m, n)=z_{1}(m, n) H_{x}^{d}\left\{z_{2}(m, n)\right\}$

if there exists a nonnegative number $\sigma<\pi$ such that

$$
\begin{aligned}
& Z_{1}\left(e^{i \omega_{x}}, e^{i \omega_{y}}\right)=0 \text { for }-\pi<\omega_{x}<-\sigma \text { and } Z_{2}\left(e^{i \omega_{x}}, e^{i \omega_{y}}\right)=0 \text { for }-\pi<\omega_{x}<\sigma<\pi \text {, or } \\
& H_{y}^{d}\left\{z_{1} z_{2}\right\}(m, n)=z_{1}(m, n) H_{y}^{d}\left\{z_{2}(m, n)\right\}
\end{aligned}
$$

if there exists a nonnegative number $\sigma<\pi$ such that

$$
Z_{1}\left(e^{i \omega_{x}}, e^{i \omega_{y}}\right)=0 \text { for }-\pi<\omega_{y}<-\sigma \text { and } Z_{2}\left(e^{i \omega_{x}}, e^{i \omega_{y}}\right)=0 \text { for }-\pi<\omega_{y}<\sigma<\pi \text {. }
$$

Theorem 3: Suppose that $z_{1}(m, n)$ and $z_{2}(m, n)$ are complex sequences with discrete-spatial Fourier transforms $Z_{1}\left(e^{i \omega_{x}}, e^{i \omega_{y}}\right)$ and $Z_{2}\left(e^{i \omega_{x}}, e^{i \omega_{y}}\right)$. Then $H_{T}^{d}\left\{z_{1} z_{2}\right\}(m, n)=z_{1}(m, n) H_{T}^{d}\left\{z_{2}(m, n)\right\}$

if there exists two nonnegative numbers $\sigma_{1}, \sigma_{2}<\pi$ such that

$$
\begin{aligned}
& Z_{1}\left(e^{i \omega_{x}}, e^{i \omega_{y}}\right)=0 \quad \text { for } \quad\left\{0<\sigma_{1}<\left|\omega_{x}\right|<\pi\right\} \cap\left\{0<\sigma_{2}<\left|\omega_{y}\right|<\pi\right\} \quad \text { and } \quad Z_{2}\left(e^{i \omega_{x}}, e^{i \omega_{y}}\right)=0 \quad \text { for } \\
& \left\{0<\left|\omega_{x}\right|<\sigma_{1}<\pi\right\} \cap\left\{0<\left|\omega_{y}\right|<\sigma_{2}<\pi\right\} .
\end{aligned}
$$

The proof is trivial.

Theorem 4: Suppose that $z_{1}(m, n)$ and $z_{2}(m, n)$ are complex sequences with discrete-spatial Fourier transforms $Z_{1}\left(e^{i \omega_{x}}, e^{i \omega_{y}}\right)$ and $Z_{2}\left(e^{i \omega_{x}}, e^{i \omega_{y}}\right)$. Then $H_{T}^{d}\left\{z_{1} z_{2}\right\}(m, n)=z_{1}(m, n) H_{T}^{d}\left\{z_{2}(m, n)\right\}$

if there exists two nonnegative numbers $\sigma_{1}, \sigma_{2}<\pi$ such that: $Z_{1}\left(e^{i \omega_{x}}, e^{i \omega_{y}}\right)=0$ for $\left\{-\pi<\omega_{x}<-\sigma_{1}\right\} \cap\left\{-\pi<\omega_{y}<-\sigma_{2}\right\}$ and $Z_{2}\left(e^{i \omega_{x}}, e^{i \omega_{y}}\right)=0$ for $\left\{-\pi<\omega_{x}<\sigma_{1}<\pi\right\} \cap\left\{-\pi<\omega_{y}<\sigma_{2}<\pi\right\}$.

The above theorems are of significance to the further applications in image processing. 


\section{Experiment, discussion and conclusions}

In this paper, we will use a texture to verify our methods. Our main aim is to verify the decomposition principles, not the reconstruction of the assisted components in this paper. On the other hand, that the discrete 2D analytic signals are rational is verified by the experiment as [6].

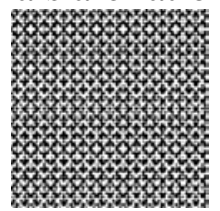

(a) The original composite multicomponent.

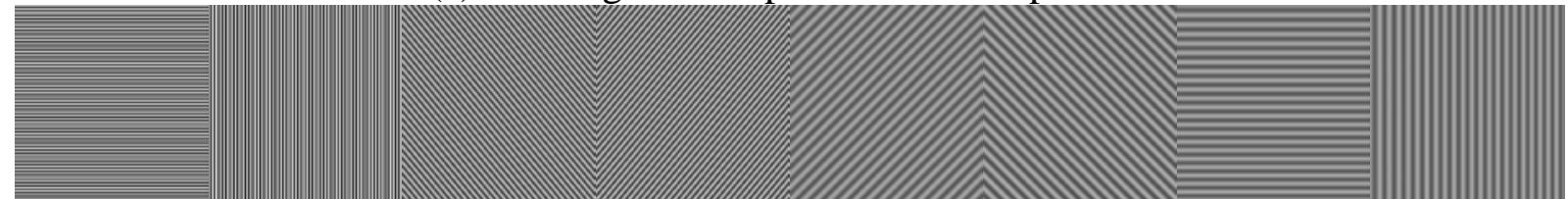

Fig.1 The decomposed monocomponents by our method.

In this paper, we investigate the discrete 2D analytic signals with some properties such as Bedrosian's principles and so on. Second, based on the derived discrete properties of discrete 2D analytic signals, we provide an iterative image decomposition method is proposed via the above principles to decompose the multicomponent image into monocomponents. Experiments on composite texture show the efficiency of the proposed methods. In our future work, we will discuss the reconstruction of assisted signals based on the original signals for image decomposition[7-9].

Acknowledgement: This work was supported by the NSFCs (61002052,61250006,61471412).

\section{References}

[1]D. Gabor, Theory of communication, IEE Proceedings 93 (1946) 429-457.

[2] Hong Li, Luoqing Lib, Tao Qian, Discrete-time analytic signals and Bedrosian product theorems,Digital Signal Processing 20 (2010) 982 - 990

[3]Stark H. An extension of the Hilbert transform product theorm. IEEE Proc, 1971, 59 :1359-1360

[4]Havlicek J P, Havlicek J W, Ngao D, etal. Skewed 2D Hilbert transforms and computed AM-FM models. IEEE Proc, 1998, 59 : 602-606

[5]Hahn L S. Multidimensional complex signals with single-orthant spectra. IEEE Proc, 1992,80 (8) : $1287-1300$

[6]Xu Guanlei, Wang Xiaotong, Xu Xiaogang, Hu jiang, Li Binyu. The Bi-Dimensional Bedrosian’s Principle for Image Decomposition. Applied Mechanics and Materials, vols. 602-205(2014). pp 3854-3858.

[7]Xu Guanlei, Wang Xiaotong, Xu Xiaogang. On Analysis of Bi-dimensional Component Decomposition via BEMD, Pattern Recognition, 2012,45(4):1617-1625

[8]G.L. Xu, X.T. Wang, X.G. Xu. Improved bi-dimensional EMD and Hilbert spectrum for the analysis of textures, Pattern Recognition, 42(5) , pp. 718-734,2009.

[9]G.L. Xu, X.T. Wang, X.G. Xu, Improved Bi-Dimensional Empirical Mode Decomposition based on 2D Assisted Signals: Analysis and Application, 2011,5(3):205-221. 